\title{
Leisure and occupational physical activity in relation to body mass index in men and women
}

\author{
Ingrid Larsson', Lauren Lissner ${ }^{2,3}$, Ingmar Näslund ${ }^{4}$ and \\ Anna Karin Lindroos'
}

Departments of 'Body Composition and Metabolism, and ${ }^{2}$ Primary Health Care, Sahlgrenska Academy at Göteborg University, Göteborg, Sweden; ${ }^{3}$ Nordic School of Public Health, Göteborg, Sweden; ${ }^{4}$ Department of Surgery,

University Hospital of Örebro, Sweden

\section{Abstract}

Background: When studying physical activity in relation to health it is important to use valid methods. Objective : To test the relative validity of a short physical activity questionnaire against 3 day physical activity records, and to describe leisure and occupational physical activity in men and women by body mass index (BMI).

Design: The validation study included 133 men and 139 women, and the cross-sectional study 1380 men and 2281 women. All subjects were aged 37-60 years, with a BMI of $17.6-59.2 \mathrm{~kg} \mathrm{~m}^{-2}$.

Results: Mean estimated physical activity level (PAL) from the 3 day activity records differed by reported grade of occupational physical activity from the short activity questionnaire $(p<0.01$ in both genders). Among men PAL values increased with increasing activity grade. The same pattern, although weaker, was seen for women. Estimated PAL values did not differ significantly by reported grade of leisure-time physical activity. Gender differences were noted in the 3 day activity records. Men recorded more sitting than women, whereas women were more engaged in walking and light activities. In the cross-sectional analysis physical activity, both in leisure time and at work, decreased with increasing degree of overweight and obesity among both men and women.

Conclusions: Mean PAL values increased with increasing grade of occupational physical activity reported in a short physical activity questionnaire. Although PAL values were not related to reported leisure-time physical activity, high BMI was associated with less physical activity both during leisure time and at work in a large group of men and women with varying BMI.

Keywords: activity record; gender difference; obesity; PAL; questionnaire

Received: 18 Jun. 2004; Revised: 9 Sep. 2004; Accepted: 13 Sep. 2004

\section{Introduction}

There is a large body of evidence, going back 50 years, that regular physical activity improves cardiovascular health (for review see ref. 1). Lack of physical activity may also be an important factor in the aetiology of obesity, and it has been suggested that decreasing levels of physical activity and a sedentary lifestyle are important factors contributing to the current obesity epidemic and its rapid distribution worldwide (2). When studying the impact of physical activity on health, it is important to use methods that are valid. The doubly labelled water (DLW) technique is today considered to be the most accurate method available for measure- ment of total energy expenditure (3); however, the method is expensive and it is not possible to study the frequency, duration and intensity of different activities. Large epidemiological studies are therefore dependent on physical activity questionnaires. Many more or less detailed physical activity questionnaires have been designed and used over the years. Although extensive questionnaires may be needed to understand further different aspects of physical activity, complicated questionnaires do not necessarily measure physical activity better than simple instruments (4).

In the Swedish Obese Subjects (SOS) study two four-point scales were used to measure leisure and 
occupational physical activity (5). The questions were originally developed for men (6) and have been used in Göteborg, Sweden, to study physical activity in relation to cardiovascular risk and mortality among men (7-9) and women (adapted version) (10). Similar questions have also been used in Norwegian (11) and Danish (12) epidemiological studies. Leisure-time physical activity has been found to be significantly associated with fitness as measured by maximal working capacity at a bicycle ergometer $(7,11)$ and maximal oxygen uptake (7); however, no study has analysed the questions in relation to physical activity level (PAL) or total energy expenditure. The first aim of the present study was therefore to test the relative validity of two physical activity questions measuring leisuretime and occupational physical activity against 3 day physical activity records. The second aim was to describe activity patterns in men and women, and leisure and occupational PAL by degree of obesity.

\section{Subjects and methods}

\section{Validation study}

Between August 1994 and February 1998, 727 subjects underwent a health examination in the SOS Reference Study (see below). At the health examination subjects were asked whether they wanted to record their physical activity during 3 days. In total, 150 men and 150 women volunteered and completed 3 day activity records. Of these, 17 men and 11 women had either no regular work or incomplete activity records and were excluded, leaving 133 men and 139 women for the analysis. Mean age \pm SD (range), body weight $(\mathrm{kg})$ and body mass index (BMI) $\left(\mathrm{kg} \mathrm{m}^{-2}\right)$ were $49.4 \pm 6.2$ (38.1$60.9), 84.2 \pm 13.2(60.4-137.6)$ and $26.0 \pm 3.6(18.6-$ 41.0) for men, and $47.8 \pm 6.1$ (37.2-60.7), 65.9 \pm 10.1 (46.0-99.8) and $24.1 \pm 3.7(17.6-38.0)$ for women, respectively. The validity of the physical activity questionnaire was tested against 3 day activity records.

\section{Cross-sectional study}

Subjects participating in the SOS Reference Study (13) and the SOS Registry Study (5) constitute the cross-sectional study group. In the SOS Reference Study 524 men and 611 women randomly selected from the communities of Göteborg and Örebro underwent a health examination between August 1994 and December 1999 (13). Mean \pm SD
BMI was $25.8 \pm 3.4 \mathrm{~kg} \mathrm{~m}^{-2}$ for men and $24.7 \pm 4.1$ $\mathrm{kg} \mathrm{m}^{-2}$ for women. In the SOS Registry Study 6328 obese men and women from all over Sweden underwent a health examination between 1987 and 2000. In the present analysis 856 men and 1670 women who underwent a health examination between August 1994 and December 1999 were included. Mean \pm SD BMI was $38.0 \pm 4.4 \mathrm{~kg} \mathrm{~m}^{-2}$ for men and $41.9 \pm 4.5 \mathrm{~kg} \mathrm{~m}^{-2}$ for women. Ages ranged from 37 to 60 years in both groups. The study groups were merged together and subjects were stratified by BMI based on the current World Health Organization (WHO) criteria for classifying normal weight, overweight and obesity (14). The BMI categories (and numbers of men/women) were 1: $18.5-24.9$ (220/370);

2: $25.0-29.9$ (250/177);

3: $30.0-34.9$ (263/82);

4: $35.0-39.9(424 / 608)$; and

5: $\geq 40.0(223 / 1044)$.

Thus, altogether 1380 men and 2281 were included in the cross-sectional analysis of leisure-time physical activity. When studying occupational physical activity 182 men and 303 women without regular work were excluded from the analysis. The subjects reported their PAL in a short questionnaire including two questions measuring leisure and occupational activity.

\section{Three day physical activity record}

Three day activity records were kept for 3 consecutive days according to a modified version of the method proposed by Bouchard et al. (15). The subjects were told to include one Saturday or Sunday and two weekdays. Every hour of the 3 days was divided into $15 \mathrm{~min}$ periods. The recording began at $06.00 \mathrm{~h}$ on the first morning and ended at $06.00 \mathrm{~h}$ on the last morning. During the registration period the subjects could choose between eight different metabolic equivalent (MET) factors representing multiples of basal metabolic rate (BMR) (16). The letter codes were: L: lying down/resting, including sleeping (MET factor 1.0); S: standing and sitting without any heavy arm work, e.g. eating, reading, watching TV, strolling (MET factor 1.4); LW: light work, e.g. doing the dishes, light housework, household shopping (MET factor 2.0); HW: heavy work, e.g. cleaning the house, wiping carpets, carrying boxes (MET factor 3.5); W: walking (MET factor 2.9); LE: low-intensity exercise, e.g. bowling, playing golf, sailing, brisk walking (MET factor 
3.2); ME: medium-intensity exercise, e.g. dancing, playing tennis, swimming, cycling (MET factor 5.3); and HE: high-intensity exercise, e.g. playing football, jogging, rowing, skiing, aerobics (MET factor 7.4). Based on the recorded time in each activity a mean individual PAL value was calculated.

\section{Self-administered physical activity questionnaire}

The questionnaire consists of two questions, one covering leisure-time physical activity and the covering occupational physical activity. The questions are

Table I. Physical activity questionnaire

(a) These questions refer to your physical activity during your leisure time during the last 12 months. If your activity varies a lot between, for instance, summer and winter, please try to average.

Please choose the group that best describes your own physical activity.

a

(one cross)

Group I: Sedentary leisure

You spend most of your leisure time reading, knitting and watching TV or film or the like

Group 2: Moderate exercise

You walk, cycle or are active in other ways, for at least 4 hours a week; for instance walking or cycling to and from your job,

Sunday walks, gardening, fishing or bowling

Group 3: Regular exercise or training

You are, for instance, engaged in running, swimming, tennis,

badminton or heavy gardening for at least 3 hours a week

Group 4: Strenuous exercise or competition

You are engaged in strenuous exercise or competition in, for

instance, running, skiing, skating, swimming, soccer or handball

regularly, at least 4 times a week

(b) These questions refer to your degree of physical activity at work during the last 12 months. We want to know how much you walk, run, climb and lift when you are at work. Below we have mentioned a few jobs as examples.

Please choose the group that best describes your own physical activity at work

\begin{tabular}{l}
\hline b \\
\hline Group 0: No paid job \\
Retired, pensioner or without a job for other reasons \\
Group I: Sedentary work \\
Most of your time you are sitting and you don't walk much at \\
work. For instance, desk workers and assembly-line workers \\
Group 2: Rather sedentary but not sitting \\
At work you walk around but you don't have to carry around \\
heavy things. For instance, housewife, shopkeeper, teacher \\
Group 3: Moderately heavy work \\
At work you walk a lot and also carry weight burdens or often \\
walk upstairs or up hills. For instance, postman, heavy industrial \\
$\quad$ worker \\
Group 4: Heavy work \\
At work you carry heavy loads and in other ways have a very heavy \\
physical job. For instance, forest work, heavy farming, fishing, \\
carrying heavy tools, house builder
\end{tabular}

graded from 1 (low physical activity) to 4 (very high physical activity). For occupational physical activity there is also an option for no work. The questionnaire is shown in Table 1.

\section{Ethical considerations}

The ethics committees of all universities in Sweden approved the protocols from the SOS study. The ethics committees of the Medical Faculty of Göteborg University, Sweden, approved the protocols from the population-based study. All subjects gave their consent to participate.

\section{Statistical analysis}

Gender-specific analyses of variance (ANOVAs) were used to test the validity of the two four-point graded physical activity questions. Individual PAL values estimated from the 3 day physical activity records were used as the dependent continuous variable and physical activity during leisure time or at work as the independent categorical variable. When the overall test was statistically significant $(p<0.05)$ Tukey's post hoc tests were applied. Grades 3 and 4 in the short questionnaire were combined in the analysis since grade 4 included very few subjects (two men and no women for leisuretime activity, and seven men and three women for occupational activity). Student's $t$-test was used to compare physical activity patterns between men and women and a logistic regression model to compare the proportion of subjects who were physically active (grades $3+4$ ) or sedentary (grade 1). Overweight (BMI category 2) and obese (BMI categories 3-5) subjects were compared with normal-weight subjects (BMI category 1). The analyses were adjusted for age and education, and results are presented as odds ratios with 95\% confidence intervals $(95 \% \mathrm{CI})$. The statistical packages SAS 8.1.0 and JMP 5.1.1 were used.

\section{Results}

In Table 2 mean PAL values estimated from the 3 day physical activity records are presented by grades of leisure and occupational physical activity from the short questionnaire. For leisure-time physical activity PAL values did not differ significantly by grade of activity in either men nor women. For occupational activity, however, mean PAL values differed significantly by activity grade in both genders. Among men, $R^{2}$ for the overall model was $27.1 \%$ and mean PAL values increased by 
Table 2. Physical activity level (PAL) from 3 day activity records $(y)$ by grades of leisure and occupational physical activity from a short physical activity questionnaire $(x)$

\begin{tabular}{|c|c|c|c|c|c|c|c|c|}
\hline & \multicolumn{4}{|c|}{ Men } & \multicolumn{4}{|c|}{ Women } \\
\hline & \multicolumn{2}{|c|}{ Leisure physical activity } & \multicolumn{2}{|c|}{ Occupational physical activity } & \multicolumn{2}{|c|}{ Leisure physical activity } & \multicolumn{2}{|c|}{ Occupational physical activity } \\
\hline & $n$ & Mean PAL & $n$ & Mean PAL & $n$ & Mean PAL & $n$ & Mean PAL \\
\hline Grade I & 9 & 1.64 & 51 & $1.50^{\mathrm{a}}$ & 13 & 1.54 & 31 & $1.53^{\mathrm{a}}$ \\
\hline Grade 2 & 90 & 1.59 & 43 & $1.63^{\mathrm{b}}$ & 104 & 1.63 & 74 & $1.63^{\mathrm{b}}$ \\
\hline Grades $3+4$ & 34 & 1.68 & 39 & $1.75^{\mathrm{c}}$ & 22 & 1.63 & 34 & $1.68^{\mathrm{b}}$ \\
\hline$p$-Value & & $=0.054$ & & $<0.001$ & & $=0.236$ & & $<0.003$ \\
\hline$R^{2}$ & & $4.4 \%$ & & $27.1 \%$ & & $2.1 \%$ & & $8.3 \%$ \\
\hline
\end{tabular}

The models are based on 133 men and 139 women in the validation group. For more detailed information on grades I-4 in the short questionnaire, see Table I.

Grades 3 and 4 were combined because there were very few subjects in grade 4.

a,b,c Significant difference between physical activity levels (ANOVA with Tukey's post hoc tests). Values in the same column with different superscript letters are significantly different $(p<0.05)$.

increasing activity grade. The same pattern was noted for women, but $R^{2}$ was lower $(8.3 \%)$ and the mean PAL value for grades $3+4$ did not differ significantly from the mean PAL value for grade 2 .

The estimated PAL value, mean \pm SD (range), from the 3 day physical activity records was $1.61 \pm$ $0.20(1.31-2.29)$ and $1.62 \pm 0.18(1.31-2.25)$ for men and women, respectively, in the validation group (not significant). Table 3 presents the time in minutes spent in the eight specified activities. Although mean PAL values were similar, gender differences in minutes spent in specific activities were observed. Men reported significantly more sitting activities than women $(p<0.01)$, whereas women reported more walking and light work $(p<$ 0.01 for both comparisons).

Fig. 1(a) shows unadjusted proportions of the 1380 men and 2281 women who reported low (grade 1) or high leisure physical activity (grades 3 or 4). In both men and women, the proportion of subjects who reported low leisure activity increased with increasing BMI category, i.e. with increasing degree of overweight ( $p$ for trend $<0.001$, both genders). Similarly, the opposite trend was observed for subjects reporting active leisure activity, i.e. the proportion of subjects who were active decreased with increasing degree of overweight ( $p$ for trend $<0.001$, both genders) (Fig. 1a). In a logistic regression model, controlling for age and education, men and women in BMI categories 3, 4 and 5 were less likely to report high leisure activity than normal-weight subjects in BMI category 1 (for details see Table 4).

Fig. 1(b) shows the unadjusted proportions of men and women who reported low (grade 1) or high occupational activity (grades 3 or 4 ). When controlling for age and education the proportions of men and women who reported low work activity were similar across the BMI categories. However, men in BMI category $5\left(\geq 40 \mathrm{~kg} \mathrm{~m}^{-2}\right)$ were more likely to report a lower work activity than

Table 3. Time $\left(\min\right.$ day $^{-1}$ ) spent in each of eight types of activity from 3 day physical activity records in 133 men and 139 women in the validation group.

\begin{tabular}{|c|c|c|c|c|c|}
\hline \multirow[b]{2}{*}{ Activity } & \multicolumn{2}{|c|}{ Men } & \multicolumn{2}{|c|}{ Women } & \multirow[b]{2}{*}{$p$} \\
\hline & Mean (SD) & Range & Mean (SD) & Range & \\
\hline Lying down & $485(59)$ & $280-680$ & $490(66)$ & $170-800$ & $=0.47$ \\
\hline Sitting & $561(186)$ & $115-1074$ & $499(158)$ & $134-945$ & $<0.01$ \\
\hline Walking & $46(50)$ & $0-285$ & $64(52)$ & $0-295$ & $<0.01$ \\
\hline Light work & 225 (178) & $0-280$ & $290(148)$ & $0-650$ & $<0.01$ \\
\hline Heavy work & 92 (128) & $0-515$ & $70(105)$ & $0-525$ & $=0.11$ \\
\hline Light exercise & $13(25)$ & $0-135$ & $10(18)$ & $0-80$ & $=0.25$ \\
\hline Medium exercise & $10(19)$ & $0-90$ & II (22) & $0-105$ & $=0.88$ \\
\hline Heavy exercise & $7(15)$ & $0-75$ & $6(12)$ & $0-40$ & $=0.65$ \\
\hline
\end{tabular}


(a)

(a)

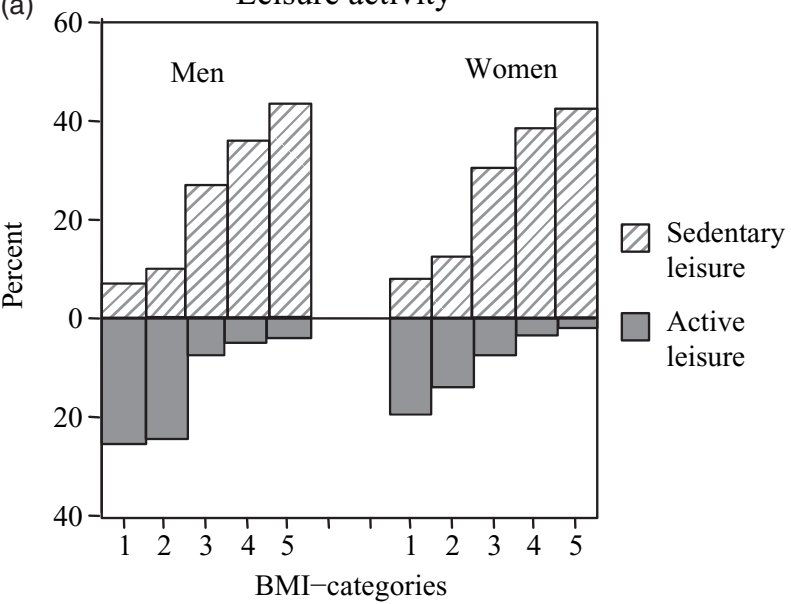

(b)

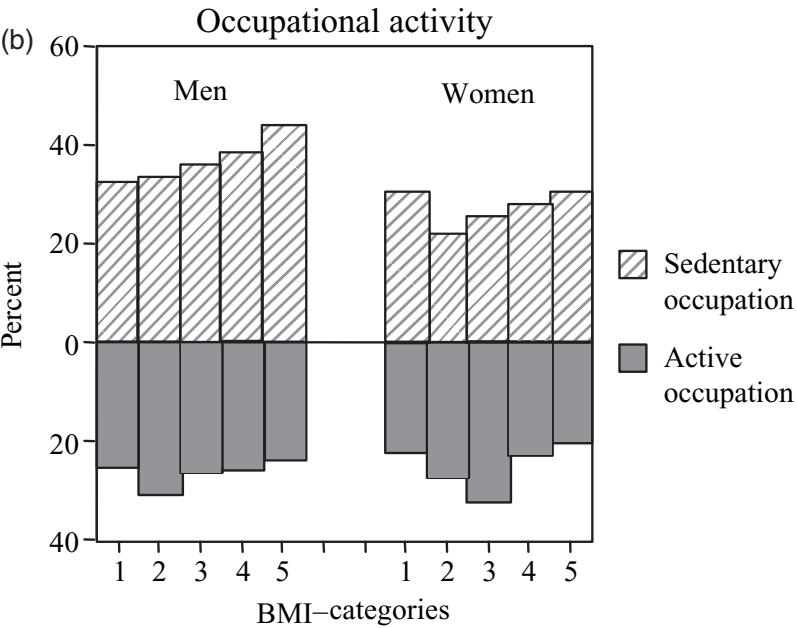

Fig. 1. (a) Leisure-time physical activity and (b) occupational physical activity by five body mass index (BMI) categories in men and women. BMI category $1:=24.9 \mathrm{~kg} \mathrm{~m}^{-2} ; 2: 25.0-29.9 \mathrm{~kg} \mathrm{~m}^{-2}$; 3: $30.0-34.9 \mathrm{~kg} \mathrm{~m}^{-2} ; 4: 35.0-39.9 \mathrm{~kg} \mathrm{~m}^{-2} ; 5: \geq 40.0 \mathrm{~kg} \mathrm{~m}^{-2}$.

normal-weight men (OR: 1.77, 95\% CI 1.16-2.71). The relationship between BMI category and high occupational activity is shown in the lower part of Fig. 1(b). The highest proportion reporting high occupational physical activity was noted in BMI category 2 for men and BMI category 3 for women, suggesting a curvilinear relationship. However, the differences were not statistically significant and when further controlling for age and education, the odds ratios for men in category 2 and women in categories 2 and 3 were not statistically significantly different to those for normal-weight men and women (Table 4). In addition, when controlling for age and education, men and women in BMI categories 4 and 5 were less likely to report a high physical activity at work than were normal-weight subjects (Table 4).

\section{Discussion}

In the present study, two simple questions on occupational and leisure physical activity were compared with 3 day physical activity records. It was found that estimated PAL values differed significantly by reported grade of occupational physical activity for both men and women. Mean PAL value also increased by increasing activity grade. For women, however, the mean PAL value for grades $3+4$ did not differ significantly from the value for grade 2. For leisure-time physical activity, PAL values did not differ significantly by grade of activity in either men nor women. Furthermore, gender differences were noted in the 3 day physical activity records. Although mean PAL values were similar, minutes spent within specific activities differed between men and women. Finally, differences in leisure-time and occupational physical activity were found between normal-weight, overweight and obese subjects. Leisure-time physical activity decreased with increasing degree of overweight and obesity and the most obese subjects were less likely to report high occupational physical activity.

Questions that grade leisure and occupational physical activity with four-point scales have previously been shown to be associated with physical fitness $(7,11)$. When physical working capacity was measured using a bicycle ergometer, Wilhelmsen et al. found an association between maximum oxygen consumption $\left(V_{\mathrm{O}_{2 \max }}\right)$ and both occupational and leisure activity, whereas physical workload capacity was only associated with leisure-time activity (7). Thune et al. also found an association between a physical workload test and leisure-time activity among women (11). In contrast to these results, the present study suggests that occupational activity is more important for total physical activity than leisure-time activity. In view of the many hours per week spent at work it is not surprising that occupational activity predicts total physical activity better than leisure activity. The associations found between physical fitness and leisure activity can be explained by the fact that cardiovascular fitness primarily reflects heavy-intensity activity rather than total energy expenditure (4). The use of cardiovascular fitness as a tool to validate total physical activity is also limited by the large individual variations in metabolic response to regular exercise due to genetic diversity (17). 
Table 4. Frequency of high reported physical activity level (grades 3 and 4 for leisure and occupational activity in the short physical activity questionnaire) in I380 men and $228 \mathrm{I}$ women in each BMI category; also shown are the odds ratio (OR) and $95 \%$ confidence interval (95\% Cl) of reporting a high physical activity level in each BMI category compared with BMI category I

\begin{tabular}{|c|c|c|c|c|c|c|}
\hline \multirow[t]{3}{*}{ BMI $\left(\mathbf{k g ~ m}^{-2}\right)$} & \multicolumn{2}{|c|}{ Men } & \multirow{3}{*}{$O R^{a}(95 \% C l)$} & \multicolumn{2}{|c|}{ Women } & \multirow{3}{*}{$O R^{a}(95 \% \mathrm{Cl})$} \\
\hline & \multicolumn{2}{|c|}{ High leisure activity } & & \multicolumn{2}{|c|}{ High leisure activity } & \\
\hline & Yes & No & & Yes & No & \\
\hline I. $18.5-24.9$ & 55 & 165 & 1.00 & 71 & 299 & 1.00 \\
\hline 2. $25.0-29.9$ & 60 & 190 & $0.99(0.65-1.5 \mathrm{I})$ & 24 & 153 & $0.80(0.48-1.34)$ \\
\hline 3. $30.0-34.9$ & 19 & 244 & $0.25(0.14-0.43)$ & 6 & 76 & $0.38(0.16-0.91)$ \\
\hline 4. $35.0-39.9$ & 21 & 403 & $0.16(0.09-0.27)$ & 21 & 587 & $0.16(0.09-0.26)$ \\
\hline 5. $\geq 40.0$ & 8 & 215 & $0.12(0.05-0.26)$ & 16 & 1028 & $0.07(0.04-0.12)$ \\
\hline \multirow[t]{2}{*}{ BMI $\left(\mathbf{k g ~ m}^{-2}\right)$} & \multicolumn{2}{|c|}{ High occupational activity } & OR $^{a}(95 \% \mathrm{CI})$ & \multicolumn{2}{|c|}{ High occupational activity } & OR $^{a}(95 \% \mathrm{Cl})$ \\
\hline & Yes & No & & Yes & No & \\
\hline I. $18.5-24.9$ & 53 & 155 & 1.00 & 81 & 281 & 1.00 \\
\hline 2. $25.0-29.9$ & 70 & 157 & $1.16(0.72-1.74)$ & 44 & 117 & I.II $(0.72-1.71)$ \\
\hline 3. $30.0-34.9$ & 60 & 168 & $0.73(0.46-1.15)$ & 23 & 48 & $1.34(0.77-2.34)$ \\
\hline 4. $35.0-39.9$ & 92 & 265 & $0.60(0.39-0.91)$ & 115 & 393 & $0.70(0.50-0.97)$ \\
\hline 5. $\geq 40.0$ & 41 & $|3|$ & $0.36(0.9 \mathrm{I}-0.95)$ & 176 & 690 & $0.57(0.42-0.78)$ \\
\hline
\end{tabular}

${ }^{a}$ Adjusted for age and education.

The four-graded question regarding occupational physical activity seemed to capture physical activity better among men than among women. Grades 3 and 4 in the four-point scale specifically concentrate on paid work that is traditionally performed by men, e.g. heavy industrial worker, forestry worker, heavy farmworker and house builder. Owing to the omission of traditional female occupational activities, also seen in other commonly used physical activity questionnaires (4), women with heavy work may underestimate their PAL. Furthermore, it is likely that more women than men work part-time and a smaller proportion of the total physical activity is therefore derived from occupational activities.

Although the present 3 day records cannot separate activities at work from home activities, gender differences were noted. Men recorded more sitting activities than women, whereas women recorded more walking and light work than men. Unfortunately, it is not possible to know whether light work recorded in this study refers to work at home or to occupational activities. Other studies also indicate gender differences in physical activity patterns $(4,18-20)$. Thus, the activity patterns of women need to be considered to obtain valid data on physical activity from women.
In the present analysis 3 day activity records were used to validate the four-point questions on leisure and occupational physical activity. Using activity records as a reference can be questioned. Activity records may not reflect habitual physical activity owing to changes in activity behaviour during the recording period. In addition, activity records may be subjected to recording bias and may thus not reflect true physical activity. The validity of physical activity records has been tested against the DLW method. These studies have shown that average energy expenditure calculated from activity records is comparable with measured energy expenditure and that subjects can be ranked by activity level. This suggests that physical activity records can be used as a valid reference method.

One problem when using activity records is the crude division of the activities and the choice of MET factors to calculate energy costs (21). The MET factors chosen for specific activities differ between studies $(15,22)$ and there may also be individual variations. Although MET factors were originally considered to be constant across a wide range of body weights (16), a recent study has shown that the energy cost of different activities expressed as multiples of BMR increases with increasing BMI (23). 
The aim of the second part of this study was to study differences in PAL among men and women with different degrees of overweight and obesity. Physical activity was reported with the two questions on leisure and occupational physical activity. In this analysis it was clearly demonstrated that the proportion of men and women who reported low leisure physical activity increased with increasing degree of obesity, whereas the opposite trend was noted for regular physical exercise during leisure time. The associations were independent of age and education and also strong among women, despite the much weaker association between the leisure activity question and activity records. Several studies have shown an inverse association between leisure-time physical activity and BMI (e.g. 18, 2426). Thus, the present study, which also included a large proportion of extremely obese subjects, confirms earlier results from population studies. Because of the cross-sectional nature of the present analysis it is not possible to make any causal inference, i.e. whether the obese subjects have become obese owing to a low PAL or whether the obese subjects are inactive owing to their obesity.

Differences in occupational physical activity by degree of obesity were less clear-cut. The proportion of men who reported high occupational activity showed a curvilinear relation between BMI group and activity level. However, when controlling for age and education, men and women with BMI $=35$ $\mathrm{kg} \mathrm{m}^{-2}$ and above were less likely to report high physical activity at work. This suggests that there is a social gradient in the relationship between occupational physical activity and degree of overweight. The relationship between BMI and occupational physical activity has been less explored than that between BMI and leisure-time physical activity. Livingstone et al. (18) found an inverse association between BMI and occupational activity, whereas Gutiérrez-Fisac et al. found no association after controlling for a number of confounding factors (26). It should be noted that the studies referred to are population based and are likely to include a very small number of subjects above BMI $35 \mathrm{~kg} \mathrm{~m}^{-2}$. The present study includes a large proportion of subjects with high BMIs and the analysis showed that the most obese subjects are less likely to report high occupational physical activity, independent of age and educational level. A possible explanation is that these extremely obese subjects are physically restrained by their obesity.

In summary, PAL estimated from 3 day activity records increased with increasing grade of reported occupational activity from a short questionnaire. Although PAL values did not differ by activity grade for leisure-time activity, high BMI was associated with less physical activity both during leisure time and at work in a large group of men and women with varying BMI.

\section{References}

1. Erlichman J, Kerbey AL, James WP. Physical activity and its impact on health outcomes. Paper 1: The impact of physical activity on cardiovascular disease and allcause mortality: an historical perspective. Obes Rev 2002; 3: 257-71.

2. Prentice A, Jebb S. Obesity in Britain: gluttony or sloth? BMJ 1995; 311: 437-9.

3. Schoeller DA, Ravussin E, Schutz Y, Acheson KJ, Baertschi P, Jéquier E. Energy expenditure by doubly labelled water: validation in humans and proposed calculation. Am J Physiol 1986; 250: R823-30.

4. Jacobs JDR, Ainsworth BE, Hartman TJ, Leon AS. A simultaneous evaluation of 10 commonly used physical activity questionnaires. Med Sci Sports Exerc 1993; 25: 81-91.

5. Kral J, Sjöström L, Sullivan M. Assessment of quality of life before and after surgery for severe obesity. Am J Clin Nutr 1992; 55: 611-4S.

6. Saltin B, Grimby G. Physiological analysis of middleaged and old former athletes. Circulation 1968; 38: 1104-15.

7. Wilhelmsen L, Tibblin G, Aurell M, Bjure J, EktrömJodal B, Grimby G. Physical activity, physical fitness and risk of myocardial infarction. Adv Cardiol 1976; 18: 217-30.

8. Rosengren A, Wilhelmsen L. Physical activity protects against coronary death and deaths from all causes in middle-aged men. Ann Epidemiol 1997; 7: 69-75.

9. Wilhelmsen L, Johansson S, Rosengren A, Wallin I, Dotevall A, Lappas G. Risk factors for cardiovascular disease during the period 1985-1995 in Göteborg, Sweden. The GOT-MONICA Project. J Intern Med 1997; 242: 199-211.

10. Lissner L, Bengtsson C, Björkelund C, Wedel $\mathrm{H}$. Physical activity levels and changes in relation to longevity. Am J Epidemiol 1996; 143: 54-62.

11. Thune I, Njølstad I, Løchen M-L, Førde OH. Physical activity improves the metabolic risk profiles in men and women. Arch Intern Med 1998; 158: 1633-40.

12. Hein HO, Suadicani P, Gyntelberg F. Physical fitness or physical activity as a predictor of ischaemic heart disease? A 17-year follow-up in the Copenhagen Male Study. J Intern Med 1992; 232: 471-9.

13. Larsson I, Berteus Forslund H, Lindroos AK, Lissner L, Näslund I, Peltonen $\mathrm{M}$ et al. Body composition in the 
SOS (Swedish Obese Subjects) reference study. Int J Obes Relat Metab Disord 2004; 28: 1317-24.

14. World Health Organization. Obesity: preventing and managing the global epidemic. Report of a WHO consultation. Geneva: WHO; 2000.

15. Bouchard C, Tremblay A, Leblanc C, Lortie G, Savard R, Theriault G. A method to assess energy expenditure in children and adults. Am J Clin Nutr 1983; 37 : 461-7.

16. FAO/WHO/UNU. Energy and protein requirements. Technical Report Series 724. Geneva: WHO; 1985.

17. Bouchard C. Individual differences in the response to regular exercise. Int J Obes Relat Metab Disord 1995; 19(Suppl 4): S5-8.

18. Livingstone MBE, Robson PJ, McCarthy S, Kiely M, Harrington K, Browne P, et al. Physical activity patterns in a nationally representative sample of adults in Ireland. Public Health Nutr 2001; 4: 1107-16.

19. Lanningham-Foster L, Nysse LJ, Levine JA. Labor saved, calories lost: the energetic impact of domestic labor-saving devices. Obes Res 2003; 11: 1178-81.

20. Buchowski MS, Acra S, Majchrzak KM, Sun M, Chen KY. Patterns of physical activity in free-living adults in the Southern United States. Eur J Clin Nutr 2004; 58: $828-37$.

21. Ainsworth BE, Haskell WL, Whitt MC, Irwin ML, Swartz AM, Strath SJ, et al. Compendium of physical activities: an update of activity codes and MET intensities. Med Sci Sports Exerc 2000; 32: S498-504.

22. Bratteby LE, Sandhagen B, Lötborn M, Samuelson G. Daily energy expenditure and physical activity assessed by an activity diary in 374 randomly selected 15 -year-old adolescents. Eur J Clin Nutr 1997; 51: 592-600.

23. Löf M, Hannestad U, Forsum E. Comparison of commonly used procedures, including the doublylabelled water technique, in the estimation of total energy expenditure of women with special reference to the significance of body fatness. Br J Nutr 2003; 90: 961-8.

24. Martinez-Gonzalez MA, Martinez JA, Hu FB, Gibney MJ, Kearney J. Physical inactivity, sedentary lifestyle and obesity in the European Union. Int $\mathbf{J}$ Obes Relat Metab Disord 1999; 23: 1192-201.

25. Norman A, Bellocco R, Vaida F, Wolk A. Total physical activity in relation to age, body mass, health and other factors in a cohort of Swedish men. Int $\mathrm{J}$ Obes Relat Metab Disord 2002; 26: 670-5.

26. Gutiérrez-Fisac JL, Guallar-Castillón P, Diez-Ganán L, Lopez García E, Banegas Banegas JR, Rodríguez Artalejo F. Work-related physical activity is not associated with body mass index and obesity. Obes Res 2002; 10: $270-6$.

\footnotetext{
Anna Karin Lindroos

Department of Body Composition and Metabolism

Sahlgrenska Academy at Göteborg University

Vita Stråket 15

SE-4I345 Göteborg

Sweden

Tel: +46313423203

Fax: +4631418527

E-mail: anna.karin.lindroos@medfak.gu.se
} 